\title{
Indexing Electron Backscatter Diffraction Patterns with a Refined Template Matching Approach
}

\author{
Alexander Foden ${ }^{1}$, David M. Collins ${ }^{2,3}$, Angus J. Wilkinson ${ }^{2}$, T. Benjamin Britton ${ }^{1}$ \\ 1. Department of Materials, Imperial College London, United Kingdom \\ 2. Department of Materials, University of Oxford, United Kingdom \\ 3. School of Metallurgy and Materials, University of Birmingham, United Kingdom
}

Electron backscatter diffraction (EBSD) is a well-established characterisation technique used to gain information about the surface of crystalline materials. An electron beam is focussed on the surface of a sample, scattering with diffraction occurs in the near surface lattice generating electrons that exit the sample over a wide range of angles. An electron backscatter pattern (EBSP) can be collected on a screen and records the angular distribution of the emitted electrons. EBSPs correspond to direct projections of the lattice planes beyond the crystal; crystal orientation measurement and phase identification relies on the property that there are constant interplanar angles, which are invariant with respect to lattice rotation $[1]$.

The indexing of EBSPs is usually done using an image processing technique called the Hough (a subset of the Radon) transform. The Hough transform converts lines seen in the pattern to points, which can be easily located by computationally methods. The interplanar angles are then found in "Hough-space" and compared to a look-up table to determine the phase and orientation of the lattice [2]. For most conventional uses, the Hough based indexing approach is sufficient, however, the Hough based indexing method often only considers the band centres, ignoring band width, intensity profile across bands and Secondary bands. This limits the accuracy and precision to how well the crystal orientation can be determined. It also limits accurate and precise measurements to be made on simple crystal structures where subtle features in the EBSP are not necessary for indexing [3].

The template matching method addresses the weaknesses of the Hough based indexing method. A library of computer generated EBSPs, of known orientation and phase is generated. The experimental EBSP to be indexed (the reference EBSP) is then compared to the library pattern by pattern, using an image cross correlation function (XCF). The XCF quantifies how similar or dissimilar two images are; a likeness metric is generated, allowing a the best match between a pattern from the library and the reference EBSP. The orientation and phase of the best match are taken to be the orientation and phase of the reference EBSP [4].

The template matching approach is usually computationally expensive. The precision and accuracy are limited by how dense or sparse the library is populated. Time can be saved by only spanning the $\mathrm{SO}(3)$ fundamental zone, such that equivalent orientations are only searched once. However, libraries still need to be large, this takes up a lot of RAM and computing time, even on powerful computers.

This current work addresses the shortfalls of existing template matching approaches, where the XCF has been updated from the usual pixel by pixel XCF, to an XCF based comparison within the Fourier domain $\mathrm{XCF}$. This has two main benefits. Firstly, lateral shift of the test pattern relative to the reference EBSP is permitted in finding the best match resulting so that a much smaller library can be used to determine the initial best fit peak. A second benefit is that the relative lateral shift is measured and can be used to 
interpolate between the discrete grid of orientations corresponding to templates. This interpolative correction step allows for much better accuracy and precision for a significant reduction in computational time. An overview of the method can be seen in figure 1.

Two main experiments are described in this work. The first uses computer generated data of known orientation and phase to test the precision and accuracy of the method. The second takes experimental data from a near perfect silicon wafer and a deformed alpha iron to test the sensitivity of the method. The results demonstrate that the new method has an accuracy in determining the crystal structure to $\sim 0.1^{\circ}$. This is comparable to the Hough based method, along with a sufficiently short computational time for indexing that it is a viable method for routine EBSD mapping measurements.

References:

[1] AJ Wilkinson and TB Britton, Mater. Today, 15 (2012), p 366.

[2] SI Wright and BL Adams, Metall. Trans. A, 23 (1992), p. 759.

[3] N Christian and K Lassen, The Technical University of Denmark (1994).

[4] YH Chen et al., Microsc. Microanal., 21 (2015), p. 739.

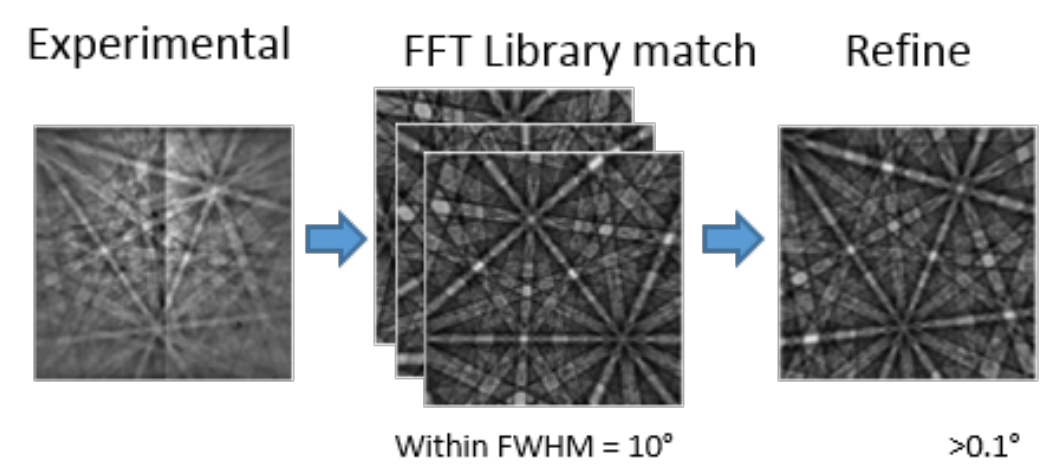

Figure 1. An overview of the new correlation method. Taking a reference image, finding a close match and performing a refinement of the initial guess. 